\title{
Myeloradiculitis with lupus-like autoimmunity following Sinovac COVID-19 vaccination
}

As we increasingly administer COVID-19 vaccination worldwide, neurological complications are to be expected. Spinal abnormalities, though rarely encountered, may result in significant morbidity requiring clinical intervention.

A 67-year old Chinese male with no past history apart from an episode of spontaneous intracranial hemorrhage 15 years ago presented with increasing bilateral lower limb weakness and numbness over 2 days, in association with low back pain. He had arrived in Singapore from Shanghai on transit to the United States, and COVID-19 PCR tests performed pre-flight were negative. He completed his second Sinovac COVID-19 vaccination (CoronaVac) 21 days prior, and was well until the current hospital admission. Specifically, there were no reports of rash, oral ulceration, joint pain, fever, headache, visual disturbances and drug usage. Clinical examination revealed that he was alert and rational. Cranial nerve and upper limb examination were unremarkable. His lower limbs were increased in tone, with the proximal right leg (MRC 3/5) slightly weaker than the left (MRC 4/5). Deep tendon reflexes were preserved bilaterally in both knees,reduced in the ankles, and Babinski responses extensor. Sensory loss to pain, touch and temperature were evident up to the knee level bilaterally. He also developed urinary retention and constipation.

MRI of the spine (Figure 1) showed two discrete segmental cord abnormality with T2 hyperintensity and swelling at T3-4 and T6-12 levels, with more diffuse cord involvement and mild enhancement at the latter, in keeping with acute thoracic myelitis. In addition, nerve root enhancement was also evident at the cauda equina.

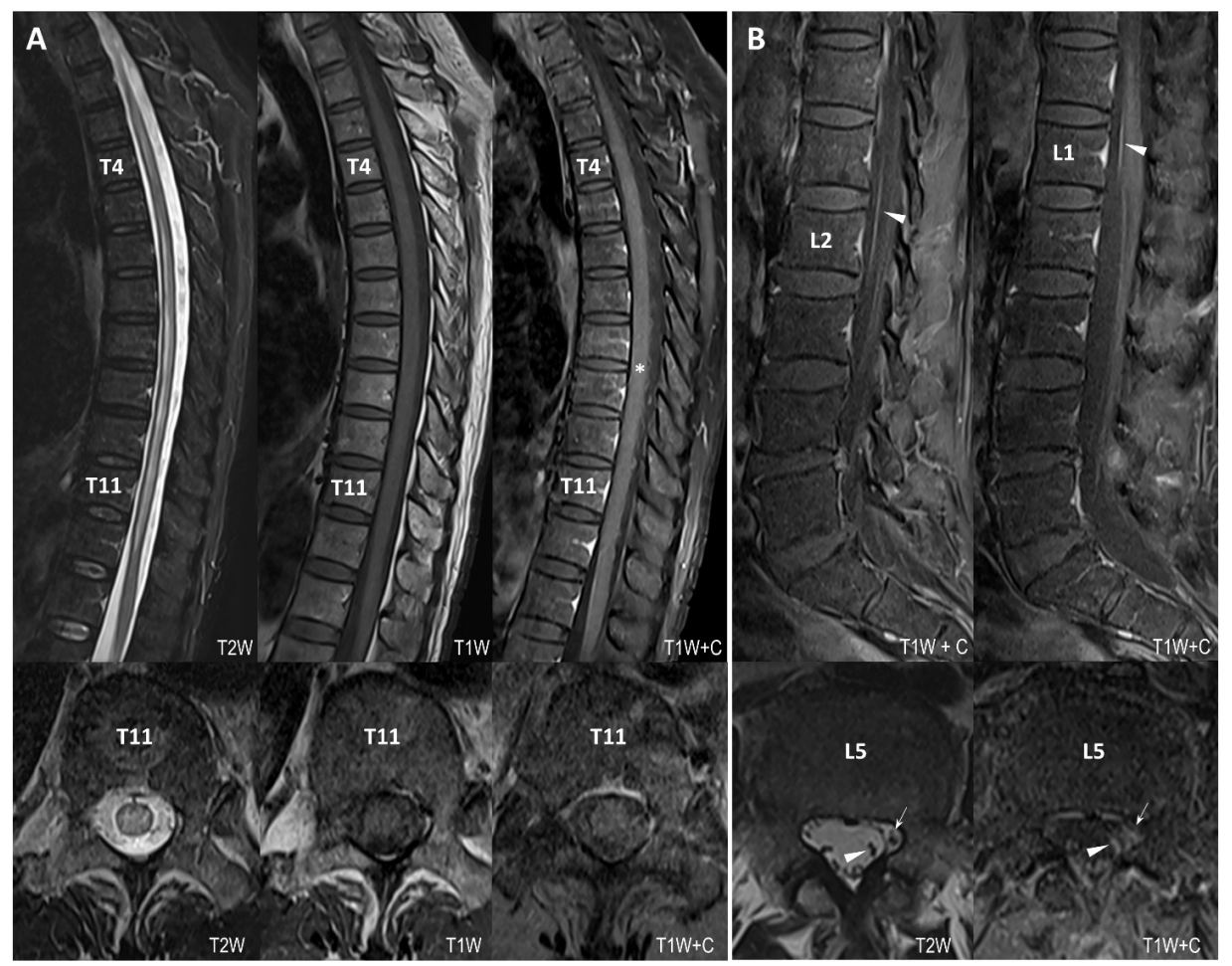

Figure 1. Sagittal (top) and axial (bottom) MRI images of the thoracic (A) and lumbar (B) spine showed segmental T2 hyperintense signals ( $1^{\text {st }}$ column$)$ in the thoracic cord, extending from T3 to T4 and T6 to T12 levels, involving both spinal grey and white matter. The shorter segment predominantly affected the right dorsolateral aspect of the cord (not shown), while the longer lower segment showed more diffuse cord signal abnormality,swelling and mild enhancement $\left(*, 3^{\text {rd }}\right.$ column). Smooth right sided lumbar nerve root enhancement was seen in the cauda equina (arrowheads) and also in the left exiting L5 (arrow) and descending $\mathrm{S} 1$ roots. 
Hematological tests showed elevated antinuclear antibody titer (> 640; normal: 160), anti-doublestranded DNA (179.87 IU; normal: < 30), mildly elevated rheumatoid factor $(261 \mathrm{U} / \mathrm{ml}$; normal $<14)$ and anti-CCP antibody $(30.5 \mathrm{U} / \mathrm{ml}$; normal < 5).Titers of serum extractable nuclear antigen antibody, anti-neutrophil cytoplasmic antibody, lupus anticoagulant and anti-cardiolipin antibodies were not elevated. 'Vitamin B12, folate levels, complement C3, C4, HIV, neurotrophic virus, hepatitis and VDRL serologies were within normal limits.Anti-aquaporin 4 and anti-myelin-oligodendrocyte panels were negative. A mild thrombocytopenia of $108 \times 10$ (9)/L recovering to $136 \times 10$ (9)/L (normal $>140$ ) was also observed.

Cerebrospinal fluid examination was suggestive of cytoalbuminologic dissociation (6 white cells, protein $0.9 \mathrm{G} / \mathrm{L}$ ) with normal cytology; PCR and serology were negative for infectious agents, including Campylobacter jejuni, and oligoclonal bands. Anti-ganglioside -antibodies, including GQ1b, GD1a, GD1b, GM1, GM2 and myelin-associated glycoprotein titers were not elevated. COVID-19 PCR tests were negative on 2 further repeats.

Lower limb nerve conduction study showed prolonged late response and $\mathrm{H}$ reflex latencies without evidence of peripheral neuropathy, in keeping with involvement of lumbar roots. He experienced significant recovery of motor power 1 week after receiving IV methyl prednisolone $1 \mathrm{gm}$ a day for 5 days.

Acute transverse myelitis has been reported in association with COVID-19 infection, occurring either as a para-infectious or post-infectious phenomenon, as well with vaccines containing viral antigens, such as the ChADOx1 nCov-19 vaccine. ${ }^{1}$ Similar occurrences of lumbar radiculitis and myeloradiculitis were also seen with other virus containing vaccines, including those for yellow fever, tick-borne encephalitis and rubella. ${ }^{2-4}$ To that end, CoronaVac, an inactivated virus vaccine, falls within this category.

Our patient had features in keeping with transverse myelitis, lumbar root and cauda equina involvement developing beyond 14 days after vaccination, suggesting a delayed autoimmune pathogenesis. ${ }^{5}$ Grossly elevated antinuclear antibody and anti-double-stranded DNA are in line with observations in lupus myelitis ${ }^{6}$ despite the lack of systemic manifestations. In addition, extensive investigations have infection, neuromyelitis optica, Guillain-Barre syndrome and multiple sclerosis as unlikely etiologies.

We report this unusual first occurrence in the light of diverse approaches to COVID-19 vaccination development, whereby a high degree of awareness of vaccine related complications focusing on timely reporting, continuous safety monitoring, and international collaboration is a necessary strategy.

${ }^{1,2}$ YL Lo, ${ }^{1} \mathrm{JJH}$ Lam, ${ }^{1} \mathrm{LL}$ Chan

${ }^{I}$ Department of Neurology, National Neuroscience Institute, Singapore General Hospital Campus; ${ }^{2}$ Duke-NUS Medical School, Singapore

Address correspondence to: Dr Lo Yew Long, National Neuroscience Institute, Singapore General Hospital Campus, Outram Road, Singapore. Email: lo.yew.long@singhealth.com.sg

https://doi.org/10.54029/2021eyv

\section{DISCLOSURE}

Financial support: None

Conflicts of interest: None

\section{REFERENCES}

1. Román GC, Gracia F, Torres A, Palacios A, Gracia K, Harris D. Acute transverse myelitis (ATM): clinical review of 43 patients with COVID-19-associated ATM and 3 post-vaccination ATM serious adverse events with the ChAdOx1 nCoV-19 vaccine (AZD1222). Front Immunol 2021; 12:653786.

2. El Nawar R, Bayle P, Girbovan A, Marque SJ, Servan J, Pico F. Meningomyeloradiculitis following yellow fever 17D vaccination: a case report. J Neurovirol 2018;24:642-6.

3. Kingston C, Zech G, Pauli C, Walker U. Lumbar radiculitis as a complication of vaccination against tick-borne encephalitis: adifferential diagnosis of low back pain and nerve root compression. Case Rep Med 2020:6130364.

4. Gilmartin RC Jr, Jabbour JT, Duenas DA. Rubella vaccine myeloradiculoneuritis. J Pediatr 1972; 80:406-12.

5. Blackburn KM, Wang C. Post-infectious neurological disorders. Therap Adv Neurol Dis 2020; 13:1-7.

6. Williams JN, Speyer CB, Kreps DJ, Kimbrough DJ, Costenbader K, Bhattacharyya S. Spinal cord syndromes in patients with systemic lupus erythematosus: differentiating lupus myelitis,neuromyelitis optica, and multiple 